\title{
Detection of branon dark matter with gamma ray telescopes
}

\author{
J. A. R. Cembranos, ${ }^{1, *}$ A. de la Cruz-Dombriz, ${ }^{2,3, \dagger}$ V. Gammaldi, ${ }^{1, *}$ and A. L. Maroto ${ }^{1, \S}$ \\ ${ }^{1}$ Departamento de Física Teórica I, Universidad Complutense de Madrid, E-28040 Madrid, Spain \\ ${ }^{2}$ Astrophysics, Cosmology and Gravity Center (ACGC), University of Cape Town, Rondebosch, 7701, South Africa \\ ${ }^{3}$ Department of Mathematics and Applied Mathematics, University of Cape Town, 7701 Rondebosch, Cape Town, South Africa
}

(Received 12 December 2011; published 7 February 2012)

\begin{abstract}
Branons are new degrees of freedom that appear in flexible brane-world models corresponding to brane fluctuations. These new fields can behave as standard weakly interacting massive particles (WIMPs) with a significant associated thermal relic density. We analyze the present constraints from their spontaneous annihilations into photons for Energetic Gamma-Ray Experiment Telescope (EGRET), Fermi-Large Area Telescope (LAT), and Major Atmospheric Gamma-Ray Imaging Cherenkov (MAGIC) telescopes, and the prospects for detection in future Cherenkov telescopes. In particular, we focus on possible signals coming from the Galactic center and different dwarf spheroidals, such as Draco, Sagittarius, Canis Major and SEGUE 1. We conclude that for those targets, present observations are below the sensitivity limits for branon detection by assuming standard dark matter distributions and no additional boost factors. However, future experiments such as the Cherenkov Telescope Array (CTA) could be able to detect gamma-ray photons coming from the annihilation of branons with masses higher than $150 \mathrm{GeV}$.
\end{abstract}

DOI: 10.1103/PhysRevD.85.043505

\section{INTRODUCTION}

Dark matter (DM) is one of the most intriguing puzzles in physics. The fact that DM cannot be made of any of the known particles is one of the most appealing arguments for the existence of new physics. The experimental search for its nature needs the interplay of new collider experiments [1] and astrophysical observations. These last ones used to be classified in direct or indirect searches (although there are other alternatives [2,3]): Elastic scattering of DM particles from nuclei should lead directly to observable nuclear recoil signatures. On the other hand, DM might be detected indirectly, by observing the products of their annihilation into Standard Model (SM) particles. We will focus our discussion on this last alternative.

It has been found that massive brane fluctuations (branons) are interesting candidates for DM in brane-world models with low tension [4]. From the point of view of the 4-dimensional effective phenomenology, massive branons are new pseudoscalar fields which can be understood as the pseudo-Goldstone bosons corresponding to the spontaneous breaking of translational invariance in the bulk space produced by the presence of the brane [5,6]. They are prevented from decaying into SM particles by parity invariance on the brane. The SM-branon low-energy effective Lagrangian $[5,6]$ can be written as

\footnotetext{
*cembra@fis.ucm.es

†alvaro.delacruzdombriz@uct.ac.za

¥vivigamm@pas.ucm.es

§maroto@fis.ucm.es
}

PACS numbers: 95.35.+d, 11.10.Kk, 12.60.-i, 98.80.Cq

$$
\begin{aligned}
\mathcal{L}_{\mathrm{Br}}= & \frac{1}{2} g^{\mu \nu} \partial_{\mu} \pi^{\alpha} \partial_{\nu} \pi^{\alpha}-\frac{1}{2} M^{2} \pi^{\alpha} \pi^{\alpha} \\
& +\frac{1}{8 f^{4}}\left(4 \partial_{\mu} \pi^{\alpha} \partial_{\nu} \pi^{\alpha}-M^{2} \pi^{\alpha} \pi^{\alpha} g_{\mu \nu}\right) T^{\mu \nu}
\end{aligned}
$$

where $\alpha=1 \ldots N$, with $N$ the number of branon species.

One can see that branons interact by pairs with the SM energy-momentum tensor $T^{\mu \nu}$ and that the coupling is suppressed by the brane tension $f^{4}$. Limits on the model parameter from tree-level processes in colliders are given by present restrictions coming from Hadron Elektron Ring Anlage, Tevatron and LEP-II, but also prospects for future colliders such as the International Linear Collider (ILC), LHC, or the Compact Linear Collider can be found in Refs. [6-9]. Additional bounds from astrophysics and cosmology were obtained in Ref. [4].

Even if branons are stable, two of them may annihilate into ordinary particles such as quarks, leptons, and gauge bosons. Their annihilation in different astrophysical objects (galactic halo, Sun, Earth, etc.) produces cosmic rays to be discriminated through distinctive signatures from the background. After branon annihilation, a cascade process would occur and in the end, the particle species that can be potentially observed would be neutrinos, gamma rays, positrons, and antimatter (antiprotons, antihelium, antideuterons, etc.) that might be detectable by means of different experimental devices. Among them, neutrinos and gamma rays have the advantage of maintaining their original direction of motion. By contrast, charged antimatter searches are hindered by the modification of the propagation trajectories.

The paper has been arranged as follows: In Section II we give a general overview for dark matter indirect detection by using gamma rays, and we present the thermal averaged cross-sections for branon scenarios. In 
Section III, we summarize the most relevant gamma-ray telescopes operating nowadays, both satellite and groundbased experiments. Section IV is devoted to the calculation of the minimum detectable fluxes from different targets by using the already-mentioned detectors. Finally, Section V contains the main conclusions about the performed analyses.

\section{GAMMA RAYS}

The differential gamma-ray flux from annihilating DM particles in galactic sources can be written as [10-12]:

$$
\begin{aligned}
\frac{\mathrm{d} \Phi_{\gamma}^{\mathrm{DM}}}{\mathrm{d} E_{\gamma}}= & \frac{1}{4 \pi M^{2}} \sum_{i}\left\langle\sigma_{i} v\right\rangle \frac{\mathrm{d} N_{\gamma}^{i}}{\mathrm{~d} E_{\gamma}} \times \frac{1}{\Delta \Omega} \\
& \times \int_{\Delta \Omega} d \Omega \int_{\text {l.o.s. }} \rho^{2}[(s)] \mathrm{d} s
\end{aligned}
$$

where the second term on the right-hand side of this equation represents the astrophysical factor, i.e., the integral of the DM mass density profile, $\rho(r)$, along the path (line of sight) between the source and the gamma-ray detector divided by the detector solid angle. On the other hand, the first term is the particle-dependent part, with $\left\langle\sigma_{i} v\right\rangle$ the thermal averaged annihilation cross-section of two DM particles into two SM particles (labeled by the subindex $i$ ). The number of photons produced in each decaying channel per energy interval $\mathrm{d} N_{\gamma}^{i} / \mathrm{d} E_{\gamma}$ involves decays and/or hadronization of unstable products such as quarks and leptons. Because of the nonperturbative QCD effects, the analytical calculation of these decay chains is a hard task to be accomplished and therefore requires Monte Carlo events generators such as PYTHIA [13] particle physics software. By using this software and after exhaustive and statistically significant simulations, the gammaray spectra were obtained in Ref. [11]. In these works, three different parametrizations were found to be able to fit all the available data in the whole accessible photon energy range for the studied channels: The first one for leptons and quarks (except for the top) is given by

$$
\begin{aligned}
x^{1.5} \frac{\mathrm{d} N_{\gamma}}{\mathrm{d} x}= & a_{1} \exp \left(-b_{1} x^{n_{1}}-b_{2} x^{n_{2}}-\frac{c_{1}}{x^{d_{1}}}+\frac{c_{2}}{x^{d_{2}}}\right) \\
& +q x^{1.5} \ln [p(1-x)] \frac{x^{2}-2 x+2}{x}
\end{aligned}
$$

the second for the top quark is encapsulated in the expression

$x^{1.5} \frac{\mathrm{d} N_{\gamma}}{\mathrm{d} x}=a_{1} \exp \left(-b_{1} x^{n_{1}}-\frac{c_{1}}{x^{d_{1}}}-\frac{c_{2}}{x^{d_{2}}}\right)\left\{\frac{\ln \left[p\left(1-x^{l}\right)\right]}{\ln p}\right\}^{q} ;$

and the last one for the $W$ and $Z$ bosons

$$
x^{1.5} \frac{\mathrm{d} N_{\gamma}}{\mathrm{d} x}=a_{1} \exp \left(-b_{1} x^{n_{1}}-\frac{c_{1}}{x^{d_{1}}}\right)\left\{\frac{\ln [p(j-x)]}{\ln p}\right\}^{q} .
$$

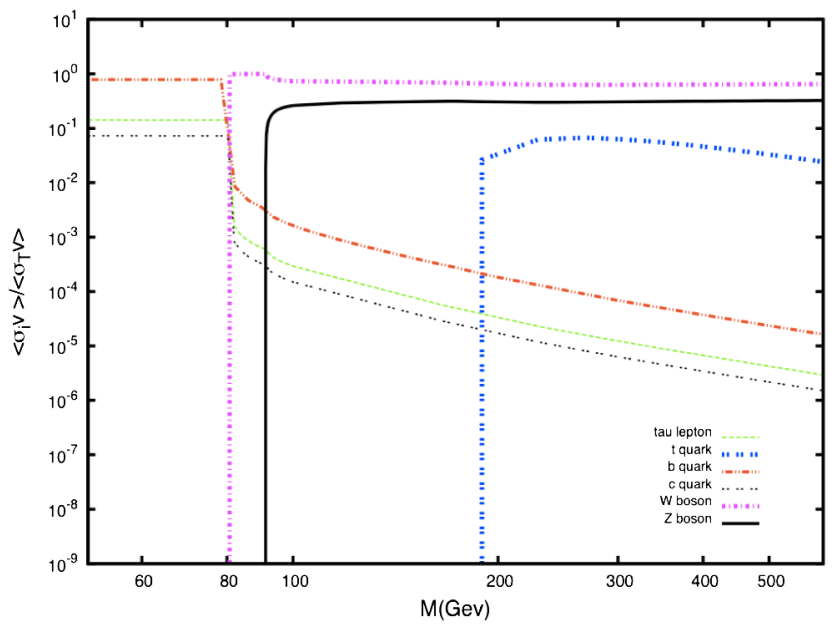

FIG. 1 (color online). Branon annihilation branching ratios into SM particles. In the case of heavy branons the main contribution to the photon flux comes from branon annihilation into $W^{+} W^{-}$and $Z Z$. The contribution from heavy fermions, i.e. annihilation into top-antitop, can be shown to be subdominant. By contrast, if $M<m_{W, Z}$, the annihilation into $W$ or $Z$ bosons is kinematically forbidden and it is necessary to take into account the rest of channels, mainly annihilation into the heaviest possible quarks.

The variable $x$ stands for $x \equiv E_{\gamma} / M$, where $E_{\gamma}$ is the photon energy and $M$ holds for the DM candidate mass. The concrete values for the constants in the above expressions (3)-(5) are given in [11] and are also available online in numerical codes [14]. The contributions of decreasing and increasing exponential factors change depending upon the channel. The asymptotic logarithmic terms appearing in expressions (3)-(5) are a consequence of the Weizsacker-Williams effect [15], although with either additive or multiplicative behavior depending on the annihilation channel. For each channel some parameters are WIMP mass dependent, whereas the remaining ones are constants.

In the case of heavy branons, the main contribution to the photon flux comes from branon annihilation into $\mathrm{ZZ}$ and $W^{+} W^{-}$(Fig. 1). The contribution from heavy fermions, i.e., annihilation into top-antitop can be shown to be subdominant [16]. In this case, the produced high-energy gamma photons could be in the range $(30 \mathrm{GeV}-10 \mathrm{TeV})$, detectable by Atmospheric Cherenkov Telescopes (ACTs) such as MAGIC $[17,18]$.

On the contrary, if $M<m_{W, Z}$, the annihilation into $W$ or $Z$ bosons is kinematically forbidden and it is necessary to take into account the rest of channels, mainly annihilation into the heaviest possible quarks [19] as can be seen in Fig. 1. In this case, the photon fluxes would be in the range detectable by space-based gamma-ray observatories [16] such as EGRET [20] and the Fermi Gamma-Ray Space Telescope spacecraft (FERMI) [21,22], with better sensitivities around $30 \mathrm{MeV}-300 \mathrm{GeV}$.

Therefore, in order to compute the final spectrum coming from branon annihilation, we need to know the total 
annihilation cross-section and its annihilation branching ratios into SM particles. For branons, the annihilation cross-sections only depend on the spin and mass of the particle, and the expressions for branons thermal average annihilations $\left\langle\sigma_{i} v\right\rangle$ in different SM particles channels, i.e., in Dirac fermions, massive gauge field, massless gauge field, and complex scalar field were calculated in Ref. [16]. For instance, the leading term for nonrelativistic branons decaying into a Dirac fermion $\psi$ with mass $m_{\psi}$, is given by

$$
\left\langle\sigma_{\psi} v\right\rangle=\frac{1}{16 \pi^{2} f^{8}} M^{2} m_{\psi}^{2}\left(M^{2}-m_{\psi}^{2}\right) \sqrt{1-\frac{m_{\psi}^{2}}{M^{2}}} .
$$

For a massive gauge field $Z$, of mass $m_{Z}$, it reads:

$$
\left\langle\sigma_{Z} v\right\rangle=\frac{1}{64 \pi^{2} f^{8}} M^{2}\left(4 M^{4}-4 M^{2} m_{Z}^{2}+3 m_{Z}^{4}\right) \sqrt{1-\frac{m_{Z}^{2}}{M^{2}}}
$$

whereas for a massless gauge field $\gamma$, the leading order is zero:

$$
\left\langle\sigma_{\gamma} v\right\rangle=0
$$

and, finally, for a (complex) scalar field $\Phi$ with mass $m_{\Phi}$ :

$$
\left\langle\sigma_{\Phi} v\right\rangle=\frac{1}{32 \pi^{2} f^{8}} M^{2}\left(2 M^{2}+m_{\Phi}^{2}\right)^{2} \sqrt{1-\frac{m_{\Phi}^{2}}{M^{2}}} .
$$

It is worth noting that there would be a gamma-ray line from direct annihilation into photons since branons couple directly to them, producing a monochromatic signal at the energy equal to the branon mass. However, this annihilation takes place in $d$-wave channel and it is highly suppressed.

\section{GAMMA-RAY TELESCOPES}

For gamma-ray detection several devices have been used in the last few years to observe galactic and extragalactic sources. Here we focus on both satellite and ground-based detectors of gamma-ray photons.

\section{A. Satellite experiments}

We will analyze the performance of two satellite telescopes: EGRET and Fermi-LAT.

\section{EGRET}

EGRET has detected gamma rays in the interval 0.02 to $30 \mathrm{GeV}$. This telescope was carefully calibrated at the Stanford Linear Accelerator Center with a quasi monoenergetic beam in the energy range of 0.02 to $10 \mathrm{GeV}$. Although the energy range was extended up to higher energies $(30 \mathrm{GeV}$ ) by using Monte Carlo simulations, we have used only data below $10 \mathrm{GeV}$ [20] due to the associated larger uncertainty at high energy. The angular resolution was energy dependent, with a $67 \%$ confinement angle of $5.5^{\circ}$ at $100 \mathrm{MeV}$, falling to $0.5^{\circ}$ at $5 \mathrm{GeV}$ on axis. The energy resolution of EGRET was 20-25\% over most of its range of sensitivity. Absolute arrival times for photons were recorded with approximately $50 \mu$ s accuracy. The overall normalization error is usually quoted as $15 \%$ [20].

\section{Fermi-LAT}

The Fermi-LAT is the principal scientific instrument on the FERMI, originally called the Gamma-Ray Large Area Space Telescope. The LAT is an imaging high-energy gamma-ray telescope covering the energy range from about $20 \mathrm{MeV}$ to more than $300 \mathrm{GeV}$ [21]. The LAT's field of view covers about $20 \%$ of the sky at any time, and it scans continuously, covering the whole sky every three hours. The LAT measures the tracks of the electron and positron that result when an incident gamma ray undergoes pairconversion, preferentially in a thin, high- $Z$ foil, and measures the energy of the subsequent electromagnetic shower that develops in the telescope calorimeter. The development of the reconstruction relies heavily on the Monte Carlo simulation of the events. The background model for this device includes cosmic rays and Earth's albedo gamma rays within the energy range $10 \mathrm{MeV}$ to $1 \mathrm{GeV}$. Particles that might either make nonastrophysical gamma rays and/or need to be rejected as background are included. The model does not include $\mathrm{X}$-rays or soft gamma rays that might cause individual detectors within the LAT to be activated [21,22]. Recent investigations [23] have looked for potential DM subhalos targets for ACTs and FERMI at energies higher than $100 \mathrm{GeV}$, claiming that multiwavelength observations could play an essential role in DM indirect searches.

\section{B. Ground-based experiments}

On the other hand, the measurement of very high-energy gamma rays using very large ground-based Cherenkov telescopes is a recent addition to the variety of experiments at the interface between particle physics, astrophysics, and cosmology.

\section{MAGIC}

In particular, the MAGIC experiment for ground-based gamma-ray astronomy is the largest of the third-generation Cherenkov telescope experiments, located at the La Palma (Canary Islands) observatory, 2,200 m above sea level. Presently, it consists of a system of two telescopes operating in a stereoscopic mode. The stereoscopic system allows for improved background rejection especially at low energies (and therefore high sensitivity), improved energy and angular resolution, and a low-energy threshold. However, in our study, we are interested in current constraints from analyses already performed with observed data. This data were collected with the initial setup (MAGIC-I) consisting of a single telescope (focal length of $17 \mathrm{~m}$ ). The threshold 
for gamma detection is around $60-70 \mathrm{GeV}$ with classical photomultiplier tubes (PMTs); future high-quantum efficiency red-extended PMTs are expected to achieve a lower threshold. MAGIC has the best light collection that has been attempted so far: the largest mirror, with an active surface of $234 \mathrm{~m}^{2}$, combined with the best available PMTs that can be obtained, of a quantum efficiency around $30 \%$. As a result, MAGIC is more sensitive to electromagnetic showers of lower energy, and does much to close the gap existing between satellite gamma-ray detectors (that can go up to $10 \mathrm{GeV}$ energy) and Cherenkov telescopes (that presently start at energies higher than $100 \mathrm{GeV}$ ). MAGIC-I has a threshold trigger energy of $\sim 50 \mathrm{GeV}$, and an analysis threshold of $\sim 70 \mathrm{GeV}$ at small zenith angle, which also permits to observe sources with higher redshift than in the past $[17,18]$.

\section{CTA}

We are also interested in estimating the prospects for future experiments as the CTA project, that is, an initiative to build the next generation of ground-based very highenergy gamma-ray instruments. Current systems of Cherenkov telescopes use at most four telescopes, providing best stereoimaging of particle cascades over a very limited area, with most cascades viewed by only two or three telescopes. An array of many tens of telescopes will allow the detection of gamma-ray-induced cascades over a large area on the ground, increasing the number of detected gamma rays dramatically, while at the same time providing a much larger number of views of each cascade. This results in both improved angular resolution and better suppression of cosmic-ray background events. The CTA will explore our Universe in depth at gamma rays of Very High Energy, i.e., $E>10 \mathrm{GeV}$ and investigate cosmic nonthermal processes, in close cooperation with observatories operating at other wavelength ranges of the electromagnetic spectrum, and those using other messengers such as cosmic rays and neutrinos. The design foresees a factor of 5-10 improvement in sensitivity in the current very high-energy gamma-ray domain of about $100 \mathrm{GeV}$ to some $10 \mathrm{TeV}$, and an extension of the accessible energy range from well below $100 \mathrm{GeV}$ to above $100 \mathrm{TeV}$ [24].

\section{ANALYSIS AND RESULTS}

Although there are other possibilities, such as the Galactic center, the best targets to search for a DM annihilation signal seems to be dwarf spheroidals (dSphs), which are the smallest known systems dominated by DM. The astrophysical part $\langle J\rangle_{\Delta \Omega}$ of the gamma-ray flux (2) of each target depends upon the DM density. This factor is not very well known and introduces the most important uncertainties in these indirect detection analyses. A classic approach uses a Navarro-Frenk-White profile. This profile is in good agreement with cold DM simulations and it allows an easy comparison with previous studies, since it has been used by many authors. For this reason, we have assumed this profile for the Draco, Sagittarius and Canis Major dSphs and for the Galactic center. In particular [25]:

$$
\rho_{\mathrm{NFW}}(r)=\frac{A}{r\left(r+r_{s}\right)^{2}},
$$

where $A$ is the overall normalization and $r_{s}$ the scale radius.

On the other hand, we have assumed an Einasto profile for SEGUE 1, the dwarf spheroidal galaxy observed with the Sloan Extension for Galactic Understanding and Exploration (SEGUE) of the Sloan Digital Sky Survey, since it is more consistent with observations [17]:

$$
\rho_{\text {Einasto }}(r)=\rho_{s} \exp \left\{-2 n\left[\left(\frac{r}{r_{s}}\right)^{1 / n}-1\right]\right\}
$$

with the scale density $\rho_{s}=1.1 \times 10^{8} M_{\odot} \mathrm{Kpc}^{-3}$, the scale radius $r_{s}=0.15 \mathrm{Kpc}$, and the index $n=3.3$ [17]. Very recent observations [26] of SEGUE 1 (considered by many authors as possibly the most DM-dominated satellite galaxy known in our galaxy) by MAGIC found no significant gamma-ray emission above the background when taking into account the spectral features of the gamma-ray spectrum of specific DM models in a supersymmetric scenario.

In any case, both the modification of the density profile and the introduction of substructures just include an additional constant in the analysis that is easy to update. With our assumptions, the values of the astrophysical factor for each source are reported in Tables I, II, III, IV, and V.

We can estimate the minimum detectable flux $\Phi_{\gamma}$ taking into account the total number of observed gamma rays. Because of the uncertainties of these kinds of analyses, it is usual to demand a significance of at least $5 \sigma$. For an observed target presenting exposure time of $t_{\exp }$ seconds, instrument of effective area $A_{\text {eff }}$ and angular acceptance $\Delta \Omega$, the significance of the detection exceeding $5 \sigma$ (or alternatively $2 \sigma$ ) is

$$
\frac{\Phi_{\gamma} \sqrt{\Delta \Omega A_{\text {eff }} t_{\text {exp }}}}{\sqrt{\Phi_{\gamma}+\Phi_{B g}}} \geq 5(2) .
$$

The DM annihilation flux $\Phi_{\gamma}$ and the background flux $\Phi_{\mathrm{Bg}}$ are given in $\mathrm{cm}^{-1} \mathrm{~s}^{-1} \mathrm{sr}^{-1}$ [25]. The evaluation of the background $\Phi_{\mathrm{Bg}}$ and its value depends both on the experiment and on the source. In the case of satellite experiments, the diffuse gamma rays flux from astrophysical sources is the only contribution to the background depending on the location of the source [25]. The background for FERMI is assumed to be the same as for EGRET [25,27,28]:

$$
\frac{\mathrm{d} N_{\mathrm{Bg}-\mathrm{a}}}{\mathrm{d} E} \approx \mathcal{N} \mathrm{GeV}^{-1} \mathrm{~cm}^{-2} \mathrm{~s}^{-1} \mathrm{sr}^{-1} \times\left(\frac{100 \mathrm{GeV}}{E_{\mathrm{Bg}}}\right)^{2.1}
$$

and the exposition time $t_{\exp }=1 \mathrm{yr}$, common for the satellite experiments. We have chosen the spectral index 2.1 
TABLE I. $\Phi_{\gamma}^{(5)}$ or $\Phi_{\gamma}^{(2)}\left(\mathrm{cm}^{-2} \mathrm{~s}^{-1} \mathrm{sr}^{-1}\right)$, where superscripts (5) and (2) denote the estimated minimum detectable flux at $5 \sigma$ or $2 \sigma$, respectively, for different detectors: EGRET, FERMI, MAGIC-I, and CTA associated with Draco. The first three are current constraints, whereas the last one is a prospect for its sensitivity. $A_{\mathrm{Eff}}\left(\mathrm{cm}^{2}\right)$ denotes the typical effective area, $\Delta \Omega$ (sr) the angular acceptance, $t_{\exp }(s)$ the exposure time, and $\Phi_{\mathrm{Bg}}\left(\mathrm{cm}^{-2} \mathrm{~s}^{-1} \mathrm{sr}^{-1}\right)$ the estimated total background flux. In order to obtain $N_{\gamma}^{(2)}\langle\sigma v\rangle / M^{2}\left(\mathrm{~cm}^{3} \mathrm{~s}^{-1}\right)$, we have used Eq. (2) with the astrophysical factor $\langle J\rangle_{\Delta \Omega}\left(10^{23} \mathrm{GeV}^{2} \mathrm{~cm}^{-5} \mathrm{sr}^{-1}\right)$ given in [25], assuming a Navarro-Frenk-White profile for the DM distribution. Most parts of the data are taken from [25], except for the values marked with a single asterisk (*) which are obtained from Ref. [27]. The $\Phi_{\mathrm{Bg}}$ signed with double asterisk ( $\left.* *\right)$ is calculated by means of Eq. (14), with $\epsilon=0.01$ for both MAGIC-I and CTA projects [25,27].

\begin{tabular}{|c|c|c|c|c|}
\hline \multicolumn{5}{|c|}{ Draco dSph } \\
\hline Experiment & EGRET & FERMI & MAGIC-I* & CTA \\
\hline$A_{\mathrm{Eff}}$ & $10^{3}(*) 1.5 \times 10^{3}$ & $10^{4}$ & $5 \times 10^{8}$ & $10^{10}$ \\
\hline$\Delta \Omega$ & $10^{-3}$ & $9 \times 10^{-5}(*) 10^{-5}$ & $10^{-5}$ & $10^{-5}$ \\
\hline$t_{\exp }$ & \multicolumn{2}{|c|}{$1 \mathrm{yr}$} & $40 \mathrm{~h}$ & $50 \mathrm{~h}$ \\
\hline$\Phi_{\mathrm{Bg}}$ & \multicolumn{2}{|c|}{$3.3 \times 10^{-7}(*) 6.7 \times 10^{-7}$} & $1.9 \times 10^{-7}(* *)$ & $\sim 10^{-7}(* *)$ \\
\hline$\Phi_{\gamma}^{(5)}$ & $1.04 \times 10^{-6}(*) 9.15 \times 10^{-7}$ & $1.14 \times 10^{-6}(*) 8.55 \times 10^{-6}$ & $1.00 \times 10^{-7}$ & $1.25 \times 10^{-8}$ \\
\hline$\Phi_{\gamma}^{(2)}$ & $2.78 \times 10^{-7}(*) 2.84 \times 10^{-7}$ & $2.97 \times 10^{-7}(*) 1.75 \times 10^{-6}$ & $3.54 \times 10^{-8}$ & $4.83 \times 10^{-9}$ \\
\hline$\langle J\rangle_{\Delta \Omega}$ & 0.1 & & 7.2 & \\
\hline$N_{\gamma}^{(5)}\langle\sigma v\rangle / M^{2}$ & $1.31 \times 10^{5}(*) 1.15 \times 10^{5}$ & $1.98 \times 10^{3}(*) 1.49 \times 10^{4}$ & $1.75 \times 10^{2}$ & 21.8 \\
\hline$N_{\gamma}^{(2)}\langle\sigma v\rangle / M^{2}$ & $3.49 \times 10^{4}(*) 3.57 \times 10^{4}$ & $5.19 \times 10^{2}(*) 3.06 \times 10^{3}$ & 61.8 & 8.42 \\
\hline
\end{tabular}

TABLE II. The same as Table I for the observation of Sagittarius. The first two columns related to EGRET and FERMI are current constraints, whereas the last one corresponding to CTA is an estimation of its possible sensitivity.

\begin{tabular}{|c|c|c|c|}
\hline \multicolumn{4}{|c|}{ Sagittarius dSph } \\
\hline Experiment & EGRET & FERMI & CTA \\
\hline$A_{\mathrm{Eff}}$ & $1.5 \times 10^{3}$ & $10^{4}$ & $10^{10}$ \\
\hline$\Delta \Omega$ & $10^{-3}$ & $\sim 10^{-5}$ & $10^{-5}$ \\
\hline$t_{\exp }$ & \multirow{2}{*}{\multicolumn{2}{|c|}{$\begin{array}{c}1 \mathrm{yr} \\
3.18 \times 10^{-6}\end{array}$}} & $100 \mathrm{~h}$ \\
\hline$\Phi_{\mathrm{Bg}}$ & & & $2.7 \times 10^{-4}(* *)$ \\
\hline$\Phi_{\gamma}^{(5)}$ & $1.59 \times 10^{-6}$ & $1.04 \times 10^{-5}$ & $1.25 \times 10^{-6}$ \\
\hline$\Phi_{\gamma}^{(2)}$ & $5.62 \times 10^{-7}$ & $2.74 \times 10^{-6}$ & $4.83 \times 10^{-9}$ \\
\hline$\langle J\rangle_{\Delta \Omega}$ & 1.3 & \multicolumn{2}{|c|}{36.9} \\
\hline$N_{\gamma}^{(5)}\langle\sigma v\rangle / M^{2}$ & $1.53 \times 10^{4}$ & $3.53 \times 10^{3}$ & 4.25 \\
\hline$N_{\gamma}^{(2)}\langle\sigma v\rangle / M^{2}$ & $5.44 \times 10^{3}$ & $9.33 \times 10^{2}$ & 1.64 \\
\hline
\end{tabular}

TABLE III. The same as Table II for the observation of Canis Major.

\begin{tabular}{lccc}
\hline \hline & \multicolumn{2}{c}{ Canis Major dSph } & \\
Experiment & EGRET & FERMI & CTA \\
\hline$A_{\text {Eff }}$ & $1.5 \times 10^{3}$ & $10^{4}$ & $10^{10}$ \\
$\Delta \Omega$ & $10^{-3}$ & $\sim 10^{-5}$ & $10^{-5}$ \\
$t_{\exp }$ & $1 \mathrm{yr}$ & & $100 \mathrm{~h}$ \\
$\Phi_{\text {Bg }}$ & \multicolumn{2}{c}{$3.87 \times 10^{-6}$} & $2.7 \times 10^{-4}(* *)$ \\
$\Phi_{\gamma}^{(5)}$ & $1.71 \times 10^{-6}$ & $1.08 \times 10^{-5}$ & $1.25 \times 10^{-8}$ \\
$\Phi_{\gamma}^{(2)}$ & $6.15 \times 10^{-7}$ & $2.94 \times 10^{-6}$ & $4.82 \times 10^{-9}$ \\
$\langle J\rangle_{\Delta \Omega}$ & 8.3 & & 139.9 \\
$N_{\gamma}^{(5)}\langle\sigma v\rangle / M^{2}$ & $2.60 \times 10^{3}$ & $9.68 \times 10^{2}$ & \\
$N_{\gamma}^{(2)}\langle\sigma v\rangle / M^{2}$ & $9.32 \times 10^{2}$ & $2.64 \times 10^{2}$ & 1.12 \\
\hline \hline
\end{tabular}


TABLE IV. The same as Table II for the observation in the direction of the Galactic center.

\begin{tabular}{|c|c|c|c|}
\hline \multicolumn{4}{|c|}{ Galactic Center } \\
\hline Experiment & EGRET & FERMI & CTA \\
\hline$A_{\mathrm{Eff}}$ & $1.5 \times 10^{3}$ & $10^{4}$ & $10^{10}$ \\
\hline$\Delta \Omega$ & $10^{-3}$ & $\sim 10^{-5}$ & $10^{-5}$ \\
\hline$t_{\exp }$ & \multirow{2}{*}{\multicolumn{2}{|c|}{$\begin{array}{c}1 \mathrm{yr} \\
1.2 \times 10^{-4}\end{array}$}} & $100 \mathrm{~h}$ \\
\hline$\Phi_{\mathrm{Bg}}$ & & & $2.7 \times 10^{-4}(* *)$ \\
\hline$\Phi_{\gamma}^{(5)}$ & $8.23 \times 10^{-6}$ & $3.51 \times 10^{-5}$ & $1.25 \times 10^{-8}$ \\
\hline$\Phi_{\gamma}^{(2)}$ & $3.23 \times 10^{-6}$ & $1.30 \times 10^{-5}$ & $4.82 \times 10^{-9}$ \\
\hline$\langle J\rangle_{\Delta \Omega}$ & 26 & & \\
\hline$N_{\gamma}^{(5)}\langle\sigma v\rangle / M^{2}$ & $3.98 \times 10^{3}$ & $1.57 \times 10^{3}$ & 0.56 \\
\hline$N_{\gamma}^{(2)}\langle\sigma v\rangle / M^{2}$ & $1.56 \times 10^{3}$ & $5.83 \times 10^{2}$ & 0.21 \\
\hline
\end{tabular}

since it is the most conservative value (see [25] or [28] for the particular values of $\mathcal{N}$ for the particular targets). In reality, the effective area of any detector depends on the particular energy at which operates. Equation (12) assumes a constant value and it is the main approximation of this equation. We will assume a typical effective area for EGRET of $1.5 \times 10^{3} \mathrm{~cm}^{2}$, whereas we will use $A_{\text {eff }}=$ $10^{4} \mathrm{~cm}^{2}$ for FERMI. By contrast, the angular acceptance is much larger for EGRET: $\Delta \Omega=10^{-3} \mathrm{sr}$, than for FERMI: $\Delta \Omega=10^{-5}$ sr [20-22,27].

In the case of ground-based experiments, besides the above diffuse gamma-ray flux, there are two other sources of background: the hadronic and the cosmic-ray electrons. In any case, the hadronic source dominates at high energies for which the ground-based experiments are sensitive. Taking into account the data observed by the Whipple $10 \mathrm{~m}$ telescope, it is possible to find a estimation for this background rate [10,27]:

$$
\begin{aligned}
\frac{\mathrm{d} \Phi_{\mathrm{Bg}-\mathrm{h}}}{\mathrm{d} E} \approx & \epsilon \times 10^{-5} \mathrm{GeV}^{-1} \mathrm{~cm}^{-2} \mathrm{~s}^{-1} \mathrm{sr}^{-1} \\
& \times\left(\frac{100 \mathrm{GeV}}{E_{\mathrm{Bg}}}\right)^{2.7},
\end{aligned}
$$

TABLE V. The same as Table I for the observation of SEGUE 1. The first column corresponds to MAGIC-I and establishes the present constraint from this target whereas the second one estimates the CTA prospects. SEGUE 1 is the only target for which we have assumed an Einasto profile for the DM distribution [17].

\begin{tabular}{ccc}
\hline \hline & SEGUE 1 & \\
Experiment & MAGIC-I & CTA \\
\hline$A_{\mathrm{Eff}}$ & $\sim 10^{8}$ & $10^{10}$ \\
$\Delta \Omega$ & $10^{-5}$ & $10^{-5}$ \\
$t_{\exp }$ & $29.4 \mathrm{~h}$ & $50 \mathrm{~h}$ \\
$\Phi_{\mathrm{Bg}}$ & $1.9 \times 10^{-7}(* *)$ & $10^{-7}$ \\
$\Phi_{\gamma}^{(5)}$ & $3.61 \times 10^{-7}$ & $1.25 \times 10^{-8}$ \\
$\Phi_{\gamma}^{(2)}$ & $1.06 \times 10^{-7}$ & $4.83 \times 10^{-9}$ \\
$\langle J\rangle_{\Delta \Omega}$ & & 11.4 \\
$N_{\gamma}^{(5)}\langle\sigma v\rangle / M^{2}$ & $3.97 \times 10^{2}$ & \\
$N_{\gamma}^{(2)}\langle\sigma v\rangle / M^{2}$ & $1.16 \times 10^{2}$ & 13.8 \\
\hline \hline
\end{tabular}

where we have integrated above the $100 \mathrm{GeV}$ threshold of MAGIC, estimating its effective area as $A_{\text {eff }}=$ $5 \times 10^{8} \mathrm{~cm}^{2}$ and its angular acceptance by $\Delta \Omega=$ $10^{-5} \mathrm{sr}$. The parameter $\epsilon$ which corresponds to the fraction of hadronic shower which is misidentified as electromagnetic is set to the order of $1 \%$ for MAGIC [17,18,27].

We are also interested in estimating the sensitivity of the next generation of ground-based Very High Energy gamma-ray CTA instruments. Although there are still many details of the CTA project to be fixed, an important improvement in the effective area is expected, thanks to the large number of telescopes in the array. We will assume a typical effective area of $A_{\text {eff }} \sim 1 \mathrm{~km}^{2}$, whereas the improvement in angular acceptance and background discrimination will be typically of order one [24]. In Tables I, II, III, IV, and V we report the technical details of each experiment, the background estimations and the resulting values of the minimum detectable gamma-ray fluxes for the Draco, Sagittarius, Canis Major dSphs, for the Galactic center and for SEGUE 1, respectively.

By using the estimated minimum detectable flux at $2 \sigma$ or $5 \sigma$ significance and the particular astrophysical factor $\left(J_{\langle\Delta \Omega\rangle}\right)$ of each target, the sensitivity on $N_{\gamma}^{(2,5)}\langle\sigma v\rangle$ has been obtained as a function of the WIMP mass depending on the particular detector. The assumption of a constant effective area gives the typical power law behavior on the WIMP mass of this sensitivity $\left(N_{\gamma}^{(2,5)}\langle\sigma v\rangle \propto M^{2}\right)$. The corresponding curves for the different targets and detectors are shown in Figs. 2 and 3. On the other hand, the theoretical value for $N_{\gamma}\langle\sigma v\rangle$ for branons has been obtained by integrating the differential spectrum $\sum_{i}\left\langle\sigma_{i} v\right\rangle \frac{\mathrm{d} N_{\gamma}^{i}}{\mathrm{~d} E_{\gamma}}$ taking into account the energy threshold of $1 \mathrm{GeV}$ for satellite experiments (Fig. 2) or $50 \mathrm{GeV}$ for ACTs (Fig. 3). The resulting $N_{\gamma}\langle\sigma v\rangle$ is a function of the two branon parameters $(f, M)$ and it does not depend on $N$ (number of branon species). This can be easily understood due to the fact that the proportionally lower flux coming from the annihilation of a larger number of branon species is compensated by the higher abundance that a larger number of species provides (for a fixed coupling, i.e. for a fixed value of $f$ ). Assuming 


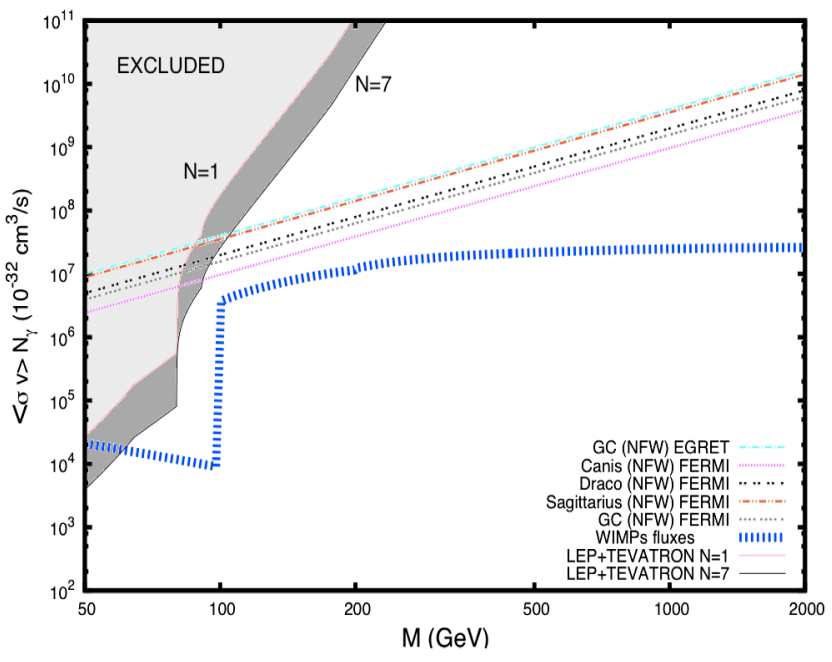

FIG. 2 (color online). Sensitivity of different targets to constrain gamma rays coming from branon annihilation. The straight lines show the estimated exclusion limits at $5 \sigma$ for satellite experiments (FERMI and EGRET). The thick dashed line corresponds to the photon flux above $1 \mathrm{GeV}$ coming from branons with the thermal abundance inside the WMAP7 [29] limits $\left(\Omega_{\mathrm{CDM}} h^{2}=0.1123 \pm 0.0035\right)$. The area on the upper left corner above the corresponding lines is excluded by LEP and Tevatron experiments for both $N=1$ and $N=7$, number of extra dimensions.

that the branon relic density agrees with WMAP observations [29], it is possible to obtain $f(M)$, which finally allows us to plot $N_{\gamma}\langle\sigma v\rangle$ as a function of the branon mass. Thus, if the integrated spectrum line is over the straight lines (which represent the sensitivity at $5 \sigma$ for a particular target), a detector will be sensitive to branon annihilation coming from a particular target. We see that present experiments (EGRET, FERMI, or MAGIC) are

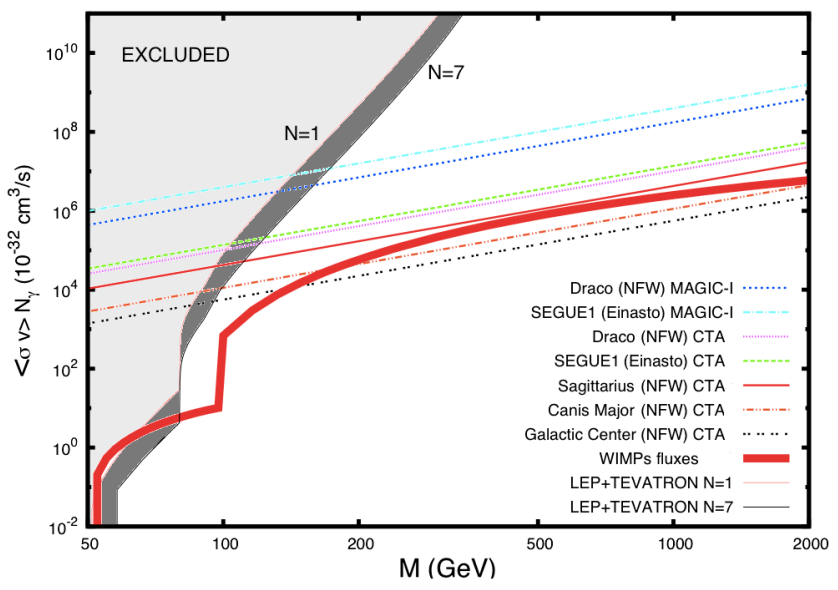

FIG. 3 (color online). Same as Fig. 2 for ground-based detectors. In this figure, the continuous thick dashed line corresponds to the photon flux above $50 \mathrm{GeV}$ coming from branons with the thermal abundance inside the WMAP7 [29] limits. unable to detect signals from branon annihilation for the targets considered. However, as shown in Fig. 3, future experiments such as CTA could be able to detect gammaray photons coming from the annihilation of branons with masses higher than $150 \mathrm{GeV}$ for observations of the Galactic center or above $200 \mathrm{GeV}$ for Canis Major.

It is important to note again that the above computations and figures are based on particular assumptions about the DM profiles and neglecting substructure contributions. Uncertainties of order one are expected for dSph satellites, but existence of boost factors of up to 3 orders of magnitude has been claimed for Galactic center analyses [30]. Even in this case, after having taken these uncertainties into account, only satellite experiments may have already observed gamma rays from branon annihilation and mainly in the low range of the spectrum. On the other hand, these signals could be reduced for core DM profiles that are in agreement for kinematic surveys not only of the Milky Way, but also of its dSphs [31].

\section{CONCLUSIONS}

We have studied the sensitivity of different gamma-ray telescopes for the observation of indirect signals of branon dark matter in brane-world scenarios. Under the assumption that branons are mass degenerate, this sensitivity depends only on two parameters of the effective theory that describes the low-energy dynamics of flexible braneworlds: the brane tension scale $f$ and the branon mass $M$.

We have computed the production of photons coming from branon annihilation happening in either some dSphs or the Galactic center and estimated the sensitivity for these cosmic photons to be detected in different experiments. In particular, we have studied the prospective detectable flux from Draco, Sagittarius, Canis Major, SEGUE 1 and for the Galactic center for EGRET, FERMI, and the future CTA. In the case of Draco and SEGUE 1, an estimation for the MAGIC telescope is given as well. The estimated constraints show that the interesting parameter space of the theory, where the thermal branon relics account for the total nonbaryonic dark matter content of the Universe, has not yet been restricted by present observation.

Concerning the next generation of ACTs, they seem to be able to prove this thermal area of the parameter space thanks to, fundamentally, the use of a large number of telescopes which can increase significantly the effective area of detection (Fig. 3). With a better sensitivity, this kind of instrument could explore the highest part of the spectrum for branons heavier than $200 \mathrm{GeV}$ by means of the observation of both dSphs (Canis Major, in particular) and the Galactic center.

Therefore, the technical instrumentation currently available appears to be insufficient to constrain the brane-world models through gamma-ray detection. On the other hand, the estimates for the next generation of 
ACTs show that these types of signals could provide the first evidences of these models. In the same Figs. 2 and 3, it is possible to see the present constraints from collider experiments. These searches are complementary and probe, in general, a different area of the parameter space of the model. Indeed, these collider analyses, in addition to direct detection experiments and other cosmic-ray studies, would be necessary to distinguish branon dark matter from other WIMP candidates since they are impossible to be distinguished from a potential positive result from gammaray observations.

\section{ACKNOWLEDGMENTS}

We would like to thank Daniel Nieto for useful comments. This work has been supported by Ministerio de Ciencia y Innovación (Spain) project numbers FIS 200801323, FPA 2008-00592, and Consolider-Ingenio Multimessenger Approach for Dark Matter Detection (MULTIDARK) CSD2009-00064. A.d.1.C.-D. also acknowledges the University Research Council, the National Research Foundation (South Africa), and Universidad Complutense de Madrid.
[1] S. Heinemeyer et al., in International Linear Collider Physics and Detector Workshop and 2nd ILC Accelerator Workshop, Snowmass, CO, 2005, (Fermi National Accelerator Laboratory, Batavia, IL, 2005); J.A.R. Cembranos, A. Rajaraman, and F. Takayama, arXiv:hep-ph/0512020; Europhys. Lett. 82, 21001 (2008); J.A.R. Cembranos, A. Dobado, and A.L. Maroto, arXiv:hep-ph/0307015; J. Phys. A 40, 6631 (2007); J. A. R. CembranosA. DobadoA. L. Maroto, in International Linear Collider Physics and Detector Workshop and 2nd ILC Accelerator Workshop, Snowmass, CO, 2005, (Fermi National Accelerator Laboratory, Batavia, IL, 2005); A. Juste et al., in International Linear Collider Physics and Detector Workshop and 2nd ILC Accelerator Workshop, Snowmass, CO, 2005, (Fermi National Accelerator Laboratory, Batavia, IL, 2005); J. A. R. Cembranos et al., arXiv:hep-ph/0603067; AIP Conf. Proc. 903, 591 (2007); ILC Collaboration, arXiv:0709.1893; ILC Collaboration, arXiv:0712.1950; ILC Collaboration, arXiv:0712.2356; T.E. Clark et al., Phys. Rev. D 78, 115004 (2008).

[2] L. Covi, J. E. Kim, and L. Roszkowski, Phys. Rev. Lett. 82, 4180 (1999); J. L. Feng, A. Rajaraman, and F. Takayama, Phys. Rev. D 68, 085018 (2003); Int. J. Mod. Phys. D 13, 2355 (2004); J. A. R. Cembranos, J. L. Feng, and L.E. Strigari, Phys. Rev. D 75, 036004 (2007); arXiv:0708.0247; arXiv:0708.0239; J.A.R. Cembranos, J. H. Montes de Oca Y., and L. Prado, J. Phys. Conf. Ser. 315, 012012 (2011); J. A. R. Cembranos, J. L. DiazCruz, and L. Prado, Phys. Rev. D 84, 083522 (2011); J. A. R. Cembranos, Phys. Rev. Lett. 102, 141301 (2009); AIP Conf. Proc. 1182, 288 (2009); Phys. Rev. D 73, 064029 (2006); AIP Conf. Proc. 1343, 604 (2011); J. Phys. Conf. Ser. 315, 012004 (2011); J. A. R. Cembranos et al., J. Cosmol. Astropart. Phys. 07 (2009) 025; T. Biswas et al., Phys. Rev. Lett. 104, 021601 (2010); J. High Energy Phys. 10 (2010) 048; Phys. Rev. D 82, 085028 (2010); A. de la Cruz-Dombriz, A. Dobado, and A. L. Maroto, Phys. Rev. D 77, 123515 (2008); Phys. Rev. Lett. 103, 179001 (2009); J. B. Jimenez et al., J. Cosmol. Astropart. Phys. 10 (2009) 029; Phys. Rev. D 80, 023004 (2009); A. Abebe et al., arXiv:1110.1191.
[3] K. Sigurdson and M. Kamionkowski, Phys. Rev. Lett. 92, 171302 (2004); S. Profumo, K. Sigurdson, P. Ullio, and M. Kamionkowski, Phys. Rev. D 71, 023518 (2005); M. Kaplinghat, Phys. Rev. D 72, 063510 (2005); J. A. R. Cembranos, J. L. Feng, A. Rajaraman, and F. Takayama, Phys. Rev. Lett. 95, 181301 (2005); L. E. Strigari, M. Kaplinghat, and J.S. Bullock, Phys. Rev. D 75, 061303 (2007).

[4] J. A. R. Cembranos, A. Dobado, and A. L. Maroto, Phys. Rev. Lett. 90, 241301 (2003); T. Kugo and K. Yoshioka, Nucl. Phys. B594, 301 (2001); J. A. R. Cembranos, A. Dobado, and A.L. Maroto, AIP Conf. Proc. 670, 235 (2003); arXiv:hep-ph/0402142; arXiv:hep-ph/0406076; arXiv:hep-ph/0411076; arXiv:astro-ph/0411262; Int. J. Mod. Phys. D 13, 2275 (2004); arXiv:astro-ph/0503622; 22nd Texas Symposium on Relativistic Astrophysics, econf C041213/1113 (2004); International Linear Collider Physics and Detector Workshop and Second ILC Accelerator Workshop, Snowmass, CO, 2005, (Fermi National Accelerator Laboratory, Batavia, IL, 2005); A. L. Maroto, Phys. Rev. D 69, 043509 (2004); 69, 101304 (2004); J.A.R. Cembranos et al., arXiv:0708.0235; J. Cosmol. Astropart. Phys. 10 (2008) 039.

[5] R. Sundrum, Phys. Rev. D 59, 085009 (1999); M. Bando et al., Phys. Rev. Lett. 83, 3601 (1999); A. Dobado and A. L. Maroto, Nucl. Phys. B592, 203 (2001); J.A.R. Cembranos, A. Dobado, and A. L. Maroto, Phys. Rev. D 65, 026005 (2001); arXiv:hep-ph/0107155.

[6] J. Alcaraz et al., Phys. Rev. D 67, 075010 (2003); J. A. R. Cembranos, A. Dobado, and A. L. Maroto, Phys. Rev. D 70, 096001 (2004).

[7] P. Achard et al., Phys. Lett. B 597, 145 (2004).

[8] P. Creminelli and A. Strumia, Nucl. Phys. B596, 125 (2001).

[9] J. A. R. Cembranos, A. Dobado, and A. L. Maroto, Phys. Rev. D 73, 035008 (2006); 73, 057303 (2006).

[10] L. Bergström, P. Ullio, and J. H. Buckley, Astropart. Phys. 9, 137 (1998).

[11] J. A. R. Cembranos, A. de la Cruz-Dombriz, A. Dobado, R. Lineros, and A. L. Maroto, Phys. Rev. D 83, 083507 (2011); AIP Conf. Proc. 1343, 595 (2011); J. Phys. Conf. 
Ser. 314, 012063 (2011); A. de la Cruz-Dombriz and V. Gammaldi, arXiv:1109.5027.

[12] J. L. Feng, K. T. Matchev, and F. Wilczek, Phys. Rev. D 63 , 045024 (2001); J.A.R. Cembranos and L.E. Strigari, Phys. Rev. D 77, 123519 (2008); J. A. R. Cembranos, J. L. Feng, and L.E. Strigari, Phys. Rev. Lett. 99, 191301 (2007).

[13] T. Sjostrand, S. Mrenna, and P. Skands, J. High Energy Phys. 05 (2006) 026.

[14] J. A. R. Cembranos, A. de la Cruz-Dombriz, A. Dobado, R. A. Lineros, and A. L. Maroto, Phys. Rev. D 83, 083507 (2011); D. Nieto, T. Hassan, and M. Doro, computer code DAMASCO, 2011.

[15] L. Bergström, T. Bringmann, M. Eriksson, and M. Gustafsson, Phys. Rev. Lett. 94, 131301 (2005); Phys. Rev. Lett. 95, 241301 (2005); A. Birkedal, K. T. Matchev, M. Perelstein et al., arXiv:hep-ph/0507194; F. Aharonian et al. (H.E.S.S. Collaboration), Phys. Rev. Lett. 97, 221102 (2006); 97, 249901(E) (2006); D. Horns (H.E.S.S. collaboration), Adv. Space Res. 41, 2024 (2008).

[16] J. A. R. Cembranos, A. Dobado, and A. L. Maroto, Phys. Rev. D 68, 103505 (2003); arXiv:astro-ph/0611911; AMS Collaboration, AMS Internal Note Report No. 2003-08-02.

[17] J. Aleksić et al. (MAGIC Collaboration), J. Cosmol. Astropart. Phys. 1106 (2011) 035.

[18] J. Albert et al., Astrophys. J. 667, 358 (2007); L. Bergström and D. Hooper, arXiv:hep-ph/0512317v2; R. Bock, The MAGIC Telescopes: Technical Details, http:// magic.mppmu.mpg.de/introduction/techdetails.html.

[19] H. U. Bengtsson, P. Salati, and J. Silk, Nucl. Phys. B346, 129 (1990).
[20] W. de Boer et al., AIP Conf. Proc. 903, 607 (2007); L. Bergström et al., J. Cosmol. Astropart. Phys. 05 (2006) 006.

[21] W.B. Atwood et al., Astrophys. J. 6971071 (2009).

[22] A. A. Abdo et al., Astrophys. J. 712147 (2010); M. L. Garde, Proc. Sci., IDM2010(2011) 116 [arXiv:astroph.HE/1102.5701v1].L. Bergström and D. Hooper, arXiv:hep-ph/0512317v2.

[23] D. Nieto et al., arXiv:1110.4744; arXiv:1109.5935.

[24] G. Maier, in Proceedings of the 31st International Cosmic Ray Conference, Łódź, Poland, 2009 (International Union of Pure and Applied Physics, London, 2009); T.C. Consortium, arXiv:1111.2183; CTA Consortium, Cherenkov Telescope Array (CTA): An Observatory for Ground-Based Gamma-Ray Astronomy, http://www.ctaobservatory.org/.

[25] N. W. Evans, F. Ferrer, and S. Sarkar, Phys. Rev. D 69, 123501 (2004).

[26] J. Aleksic et al., arXiv:1109.6781.

[27] L. Bergström and D. Hooper, Phys. Rev. D 73, 063510 (2006).

[28] N. Gehrels and P. Newman, The Energetic Gamma Ray Experiment Telescope (EGRET), http://heasarc.gsfc .nasa.gov/docs/cgro/egret/.

[29] E. Komatsu et al. (WMAP Collaboration), Astrophys. J. Suppl. Ser. 192, 18 (2011).

[30] F. Prada, A. Klypin, J. Flix Molina, M. Martínez, and E. Simonneau, Phys. Rev. Lett. 93, 241301 (2004).

[31] P. Salucci et al., arXiv:1111.1165. 